Supporting information for:

\title{
Direct Evidence of Solution-Mediated Superoxide Transport and Organic Radical Formation in Sodium- Oxygen Batteries
}

\author{
Chun Xia ${ }^{\dagger \ddagger}$, Russel Fernandes ${ }^{\dagger \ddagger}$, Franklin H. Cho ${ }^{\S 凶}$, Niranjan Sudhakar ${ }^{\ddagger}$, Brandon

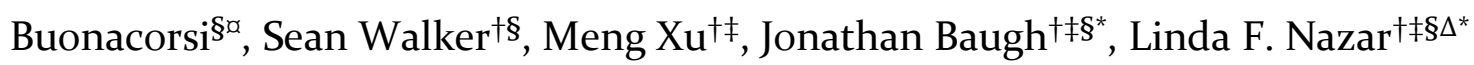 \\ $\dagger$ Department of Chemistry, University of Waterloo, Waterloo Ontario N2L 3G1, Canada \\ \$Waterloo Institute for Nanotechnology, University of Waterloo, Waterloo Ontario N2L 3G1, Canada \\ $\S$ Institute for Quantum Computing, University of Waterloo, Waterloo, Ontario N2L 3G1, Canada \\ aDepartment of Physics and Astronomy, University of Waterloo, Waterloo, Ontario N2L 3G1, Canada \\ *Email: baugh@uwaterloo.ca, lfnazar@uwaterloo.ca
}




\section{Experimental}

Purification of electrolyte: Diethylene glycol dimethyl ether (Diglyme, anhydrous grade, 99.5\%, SigmaAldrich) was distilled over calcium hydride (reagent grade, $95 \%$, Sigma-Aldrich) under a nitrogen atmosphere and stored over molecular sieves (4 A, beads 8-12 mesh, Sigma Aldrich) for one week prior to being used as solvent. Sodium triflate (NaOTf, $99.5 \%$ purity, Solvionic) was vacuum-dried at $150{ }^{\circ} \mathrm{C}$ overnight using a buchi glass oven. An electrolyte solution of $0.5 \mathrm{M}$ sodium triflate in diglyme was prepared in an argon-filled glove box $\left(<0.5 \mathrm{ppm} \mathrm{H}_{2} \mathrm{O}\right.$ and $\left.<1.0 \mathrm{ppm}_{2}\right)$. The water content of electrolyte was approximately 10 ppm, as determined by a Mettler Toledo C30 Karl Fischer (KF) titrator. When needed, different amounts of deionized water were added into the electrolyte and its precise water concentration was determined by KF titration before use.

Preparation of spin trap solution: 5, 5-dimethyl-1-pyrroline-N-oxide (DMPO) was used as the spin trap to capture radicals in the electrolyte. The spin trap solution consisted of 0.1 M DMPO in dimethyl sulfoxide (DMSO) and was prepared in an argon-filled glove box. DMPO (GC grade, $\geq 98.0 \%$, Sigma-Aldrich) was used as received and DMSO (GC grade, $>99.5 \%$, Sigma-Aldrich) was distilled over calcium hydride under reduced pressure.

Cell assembly using a bulk cell design: A home-designed $\mathrm{Na}^{-} \mathrm{O}_{2}$ glass cell was used for our studies. The assembly of the glass cell was carried out in an argon-filled glovebox. The cell was composed of a $20 \mathrm{~mL}$ glass vial (the vessel for the cell), and a modified rubber stopper. The modified rubber stopper possessed a $1 \mathrm{~mL}$ syringe and two stainless steel wires with alligator clips soldered to the bottom of the wires. The gas diffusion layer cathode (GDL H2315, Freudenberg) and sodium anode (ACS Reagent, Sigma-Aldrich) were clamped by the alligator clips to ensure good electrical contact. The needle of the syringe was installed into the rubber stopper and its tip was in close proximity to the GDL. This allows for effective collection of the soluble reaction intermediates and products. Prior to measurements, the glass vial was filled with $4.5 \mathrm{~mL}$ of electrolyte, plugged with the modified rubber stopper containing both anode and cathode, transferred into an oxygen-filled glove bag (AtmosBag, Sigma-Aldrich), and purged with high purity oxygen (5.0 research grade). Before assembly and measurements, the cathodes were vacuum-dried at $300^{\circ} \mathrm{C}$ to remove any trace amounts of $\mathrm{H}_{2} \mathrm{O}$.

Galvanostatic cycling: In order to avoid the influences from air and moisture, the electrochemical measurements were carried out in a nitrogen-filled glove bag. Unless otherwise stated, a constant current of $50 \mu \mathrm{A} \mathrm{cm}^{-2}$ was applied for both discharge and charge. Pristine cathodes were used for discharge studies while cathodes pre-discharged to $2 \mathrm{mAh} \mathrm{cm}^{-2}$ were used for charge studies. All the cells were rested at open circuit conditions for $2 \mathrm{~h}$ prior to measurements to attain equilibrium. 
Characterization: Electron spin resonance (ESR) spectroscopy was used to study the radicals present in the electrolyte. For each measurement, $100 \mu \mathrm{L}$ of electrolyte was extracted from the $\mathrm{Na}-\mathrm{O}_{2}$ glass cell through the needle of the syringe without interruption to the ongoing electrochemical reactions. The extracted electrolyte was then mixed with $500 \mu \mathrm{L}$ of $0.1 \mathrm{M} \mathrm{DMPO} / \mathrm{DMSO}$ solution. $100 \mu \mathrm{L}$ of the resulting solution was immediately transferred into a capillary tube $(4 \mathrm{~mm}$ thin wall precision quartz, WilmadLabGlass) and sealed with a glass-gas inlet stopper for continuous-wave (CW) ESR measurements, and immediately cooled to $210 \mathrm{~K}$.

CW ESR spectra were collected using a Bruker X-band EMXmicro equipped with EMX standard resonator and ESR900 continuous flow cryostat (Oxford Instruments) for temperature control. Typical conditions of the ESR experiments were as follows: microwave frequency of $9.39 \mathrm{GHz}$, incident microwave power of $0.63 \mathrm{~mW}$, modulation field strength of $1 \mathrm{G}$ with averaging 32 scans or $2 \mathrm{G}$ with averaging 16 scans. We noticed that cooling down the samples with liquid helium was necessary to eliminate the degradation of DMPO-OOH adduct in the electrolyte and to tune the ESR cavity. Unless noted otherwise, ESR measurements were carried at $210 \mathrm{~K}$.

The morphology changes of cathodic products at different discharge/charge times and as a function of $\mathrm{H}_{2} \mathrm{O}$ content were investigated using a LEO 1530 field-emission scanning electron microscope (SEM) equipped with an energy-dispersive X-ray spectroscope (EDX) attachment (Zeiss). In order to avoid the precipitation of superoxide due to the evaporation of electrolyte, the discharged/charged cathodes were taken from the glass cell immediately once the polarization was halted. Then the cathodes were washed with dried diglyme for $5 \mathrm{~s}$ to remove remaining electrolyte. Lastly, after vacuum-drying for $30 \mathrm{~min}$, cathodes were transferred into a gastight sample holder for SEM measurements.

Powder X-ray diffraction (XRD) patterns were collected using a Bruker D8-Advance (Cu-K 1 radiation; $\lambda=1.5405 \AA$ ). The XRD patterns of cathodes were performed in air using a gastight sample holder with a Kapton film window.

For ${ }^{1} \mathrm{H}$ nuclear magnetic resonance $\left({ }^{1} \mathrm{H}-\mathrm{NMR}\right)$ analysis of by-products, the cathodes were immersed in $700 \mu \mathrm{L}$ of deuterium oxide ( $\mathrm{D}_{2} \mathrm{O}, 99.9 \%$, Sigma-Aldrich) for $30 \mathrm{~min}$. NMR studies were conducted on the resulting solution on a high-resolution NMR spectrometer (Bruker $300 \mathrm{MHz}$ ) at room temperature. ${ }^{1} \mathrm{H}$ spectrum in $\mathrm{D}_{2} \mathrm{O}$ was referenced to the solvent residual peak at $4.79 \mathrm{ppm}$. 


\section{Detailed discussion of Figure 6 (effect of charging time on electrolyte samples and water concentration on the ESR spectra of electrolyte samples).}

The sample with either longer charge time or higher $\mathrm{H}_{2} \mathrm{O}$ concentration in the electrolyte presents a distorted ESR pattern compared with that of DMPO-OOH radicals, which indicates another radical species is formed under these conditions. Subtraction of the two measured spectra as shown in Figure $6 \mathrm{a}$ and $\mathrm{b}$ suggests that the ESR pattern of the newly formed radicals to be six equally distanced peaks, which can be observed when $\alpha_{N} \approx 2 \alpha_{H}$. The simulated ESR spectra, following similar procedures described in the section below entitled "Figure S2: Confirmation of DMPO-OOH radicals by ESR spectroscopy, " with simulation parameters of $\mathrm{g}=2.0084, \alpha_{N}=12.8 \mathrm{G}, \alpha_{H}=6.4 \mathrm{G}, \Gamma_{\text {Lorentzian, } \mathrm{p}-\mathrm{p}}=3.1 \mathrm{G}$ for Figure $6 \mathrm{a}$ and $\mathrm{g}=2.0083, \alpha_{N}=12.6$ $\mathrm{G}, \alpha_{H}=6.7 \mathrm{G}, \Gamma_{\text {Lorentzian, } \mathrm{p}-\mathrm{p}}=3.3 \mathrm{G}$ for Figure $6 \mathrm{~b}$ (no hyperfine coupling to the second hydrogen nucleus spin), show good agreement with the subtracted spectra. The hyperfine coupling strengths obtained from the simulations suggest that the paramagnetic species responsible for the observed signals are likely alkyl oxygen-centered organic radicals (RO), such as $\mathrm{CH}_{3} \mathrm{O}^{*}\left(\alpha_{N}=13.6 \mathrm{G}, \alpha_{H}=7.6 \mathrm{G}\right), \mathrm{CH}_{3} \mathrm{CH}_{2} \mathrm{O}^{*}\left(\alpha_{N}=13.2 \mathrm{G}\right.$, $\left.\alpha_{H}=7.0 \mathrm{G}\right)$, and $\mathrm{CH}_{3} \mathrm{CH}_{2} \mathrm{CH}_{2} \mathrm{CH}_{2} \mathrm{O}^{*}\left(\alpha_{N}=13.6 \mathrm{G}, \alpha_{H}=6.8 \mathrm{G}\right) .{ }^{1}$ In comparison with the carbon-centered radicals formed under reduction conditions (see Figure S5), it seems that the formation of oxygen-centered radicals are preferred under oxidation conditions, indicating a different decomposition mechanism.

Consistent with the results here, Adams et al. recently proposed that the decomposition of glyme-based solvents could also undergo $\mathrm{H}$-abstraction followed by a $\beta$-scission or superoxide nucleophilic attack. This may lead to the formation of alkyl oxygen radicals, e.g., $\mathrm{CH}_{3} \mathrm{O} \cdot{ }^{2}$ However, due to only limited numbers of $\mathrm{RO}^{\bullet}$ being studied, it is difficult to identify whether these are primary or secondary radicals. 


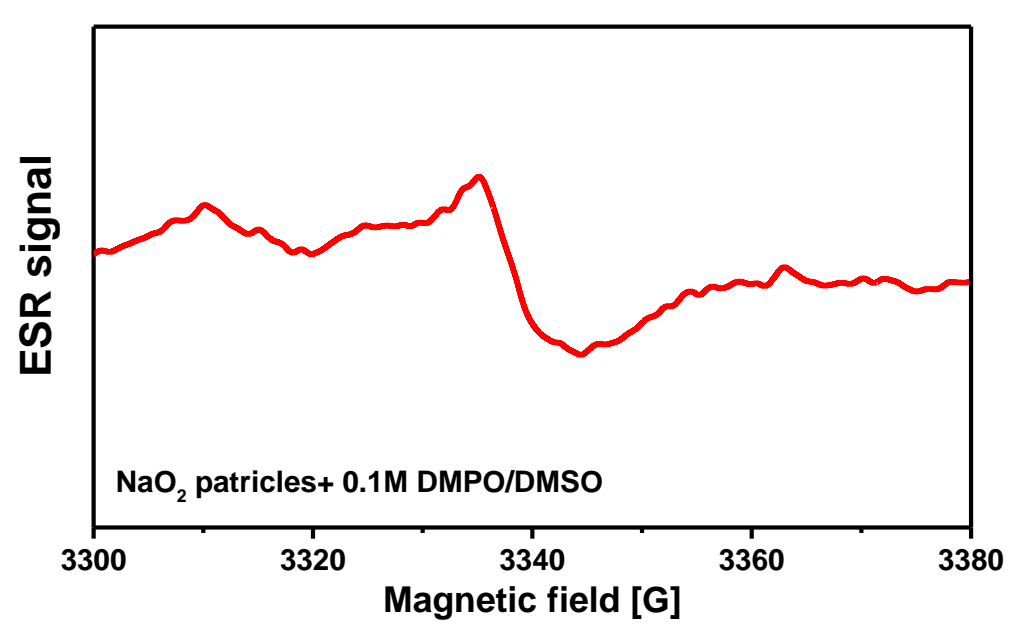

Figure S1. ESR spectrum obtained after immersing a discharged cathode containing solid $\mathrm{NaO}_{2}$ crystals in $0.1 \mathrm{M}$ DMPO/DMSO for $10 \mathrm{~s}$.

Discussion of Figure S1 (non-reactivity of DMPO with solid $\mathrm{NaO}_{2}$ particles). The discharged cathode with solid $\mathrm{NaO}_{2}$ product was mixed with a DMPO/DMSO solution for approximately 10 seconds. The resulting solution was then transferred into a quartz tube for ESR measurement at $220 \mathrm{~K}$. As shown in Figure S1, the obtained ESR spectrum shows two broad peaks, which is different from the four peak pattern of the DMPO-OOH adduct as presented in Figure S3. This indicates that solid $\mathrm{NaO}_{2}$ particles do not readily react with DMPO in DMSO solution to form the DMPO-OOH adduct. The observed ESR signal is likely due to the paramagnetic $\mathrm{NaO}_{2}$ particles and carbon fibers that remained in the ESR sample after the cathode was removed. ${ }^{3}$ 


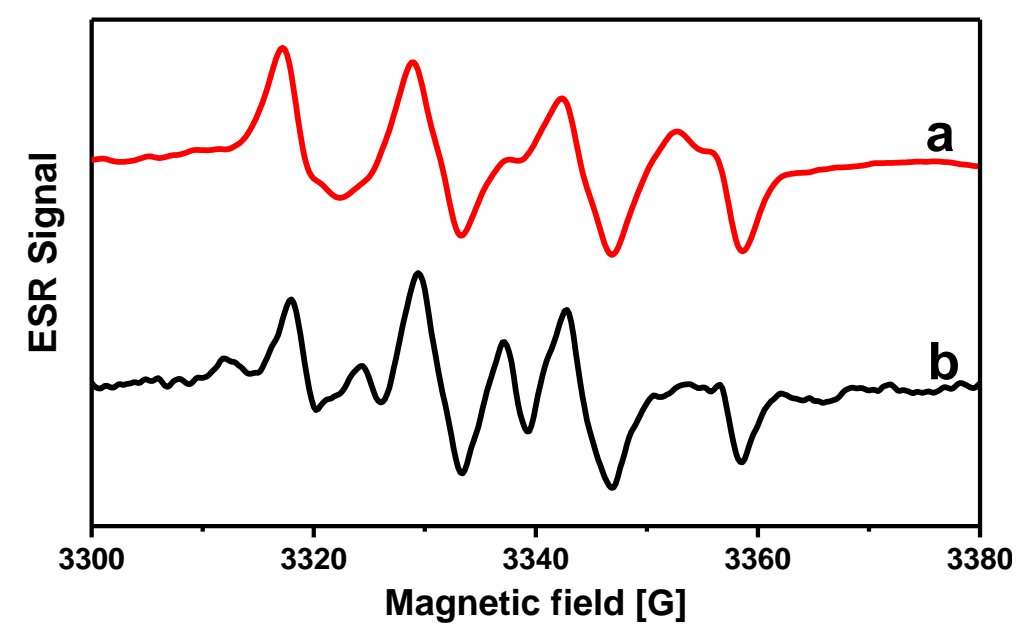

Figure S2. ESR spectra of $3 \mathrm{mM} \mathrm{KO} / \mathrm{DMSO}$ mixed with $0.1 \mathrm{M} \mathrm{DMPO/DMSO}$ for $10 \mathrm{~s} \mathrm{(a)} \mathrm{and} 12 \mathrm{~h}$ (b).

Discussion of Figure S2 (metastable DMPO-OOH adducts). Potassium superoxide $\left(3 \mathrm{mM} \mathrm{KO}_{2}\right)$ in DMSO solution was prepared by dissolving commercial $\mathrm{KO}_{2}$ (Aldrich) in distilled DMSO solvent under an argon atmosphere. Then $100 \mu \mathrm{L}$ of the resulting solution was mixed with $500 \mu \mathrm{L}$ of $0.1 \mathrm{M}$ DMPO/DMSO for $10 \mathrm{~s}$ in a nitrogen-filled glove bag. $100 \mu \mathrm{L}$ of the mixture was then immediately transferred into an ESR tube and sealed with a glass-gas inlet stopper for CW ESR measurement. The mixture was measured again after a rest period of $12 \mathrm{~h}$ at room temperature. As shown in Figure S2 (curve a), the ESR spectrum of the quickly mixed sample shows the typical four-peak pattern of the DMPO-OOH adduct (details are given in Figure S3). However, additional ESR peaks appear when the mixture is measured again after sitting at room temperature for $12 \mathrm{~h}$ (see Figure S2 (curve b)). As reported in the literature, the metastable DMPO-OOH adduct slowly decays to the more stable DMPO-OH adduct and 5,5-Dimethyl-2-pyrrolidone-N-oxyl (DMPOX), which may generate these additional ESR peaks. ${ }^{4}$ To avoid these undesired side reactions, all the electrolyte samples were mixed with DMPO at room temperature for $10 \mathrm{~s}$ and ESR measurements were subsequently carried out at a low temperature of $210-240 \mathrm{~K}$. 


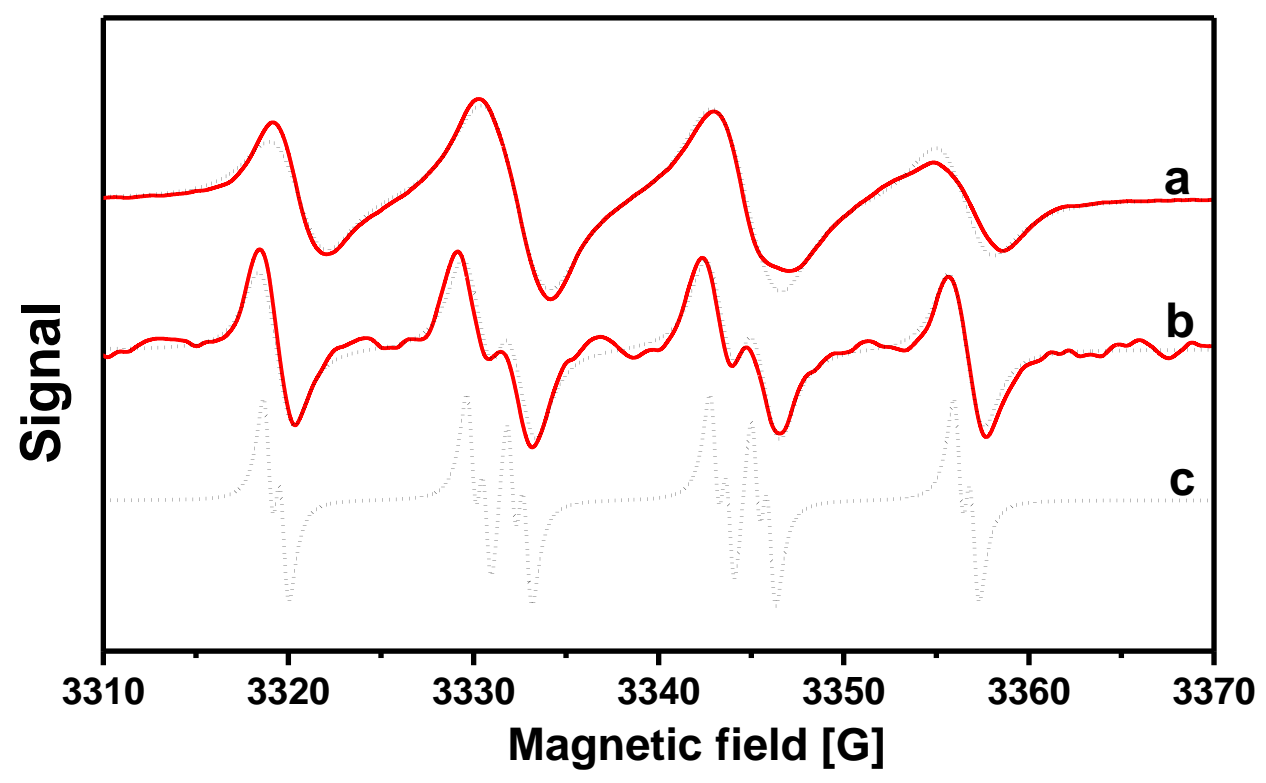

Figure S3. CW ESR spectra of 60 min discharged electrolyte measured at $210 \mathrm{~K}$ (a) and $240 \mathrm{~K}$ (b). Solid red lines and dashed black lines indicate measured and simulated spectra, respectively. Parameters used in the simulations are: $\mathrm{g}=2.0085, \alpha_{N}=12.5 \mathrm{G}, \alpha_{H}^{\beta}=11.1 \mathrm{G}, \alpha_{H}^{\gamma}=1.2 \mathrm{G}, \Gamma_{\text {Lorentzian, } \mathrm{p}-\mathrm{p}}=2.5 \mathrm{G}$ for (a) and $\mathrm{g}=2.0084, \alpha_{N}=13.2 \mathrm{G}, \alpha_{H}^{\beta}=11.0 \mathrm{G}, \alpha_{H}^{\gamma}=0.8 \mathrm{G}, \Gamma_{\text {Lorentzian,p-p }}=0.7 \mathrm{G}$ for $(\mathrm{b})$. The ESR spectra reveal that the paramagnetic species present in the electrolyte samples are the DMPO-OOH radicals. Fine structure of DMPO-OOH radicals from a weak hyperfine coupling to a second hydrogen nucleus $\left(\alpha_{H}^{\gamma}\right)$ were not resolved in the measured spectra due to linewidth broadening. (c) Simulated spectrum using the same Hamiltonian parameters as (b) except $\Gamma_{\text {Lorentzian, }-\mathrm{p}}=0.07 \mathrm{G}$ which shows the expected fine structures. For ease of comparison, each spectrum is plotted with separate normalization.

Discussion of Figure S3 (confirmation of DMPO-OOH adducts by ESR spectroscopy). Figure S3a and b show CW ESR spectra of electrolyte samples discharged for 60 min and measured at 210 and $240 \mathrm{~K}$, respectively. Similar to the DMPO-OOH adduct, the DMPO-OH adduct also shows an ESR signal composed of a 4 peak pattern. ${ }^{5}$ The major difference in ESR spectra between DMPO-OH and DMPO-OOH radicals is that the unpaired electrons in DMPO-OH radicals exhibit hyperfine coupling to one nitrogen $(\mathrm{N})$ and one hydrogen $(\mathrm{H})$ nucleus, and their strengths of hyperfine coupling $\left(\alpha_{N}\right.$ and $\left.\alpha_{H}\right)$ are identical $\left(\alpha_{N}=\alpha_{H}=14.9 \mathrm{G}\right)$. This gives rise to the well-known four peak pattern with 1:2:2:1 intensity ratio at equal spectral linewidth $(\Gamma) .{ }^{5}$ However, for DMPO-OOH radicals, the observed strength of $\alpha_{H}^{\beta}$ is smaller than that of $\alpha_{N}\left(\alpha_{N}=13-14 \mathrm{G}\right.$ and $\alpha_{H}^{\beta}=10-11 \mathrm{G}$; slight variations among different solvents have been reported), ${ }^{6}$ which results in further splitting of the central two peaks by the difference of $\alpha_{N}$ and $\alpha_{H}^{\beta}$. As seen in 
Figure S3b, this splitting was clearly observed in the spectrum of the electrolyte at $240 \mathrm{~K}$, indicating that the radicals responsible for the observed ESR signals are DMPO-OOH adducts. However, the spectrum at $210 \mathrm{~K}$ shows linewidth broadening which obstructs the observation of the lineshape splitting (see Figure S3a). Without the splitting feature of the central two peaks arising from $\alpha_{N} \neq \alpha_{H}^{\beta}$, the the spectral shape looks qualitatively similar to that of DMPO-OH radicals. The linewidths of the middle two peaks are larger than those of the outer two peaks, however, indicating an overlapping lineshape from unresolved splitting, and showing that the observed ESR signals are indeed from the DMPO-OOH radicals. The cause of the linewidth broadening is most likely due to slow movement of the radicals in the solvent at lower temperatures. However, since the measurement temperature is still higher than the freezing point of diglyme $(209 \mathrm{~K})$, the radicals are still in the fast-motional/isotropic regime where they do not exhibit noticeable slow-motional/rigid limit characteristics. ${ }^{7}$

In addition, it has been reported that room temperature spectra of DMPO-OOH show a weak hyperfine coupling to another $\mathrm{H}$ nucleus $\left(\alpha_{H}^{\gamma} \sim 1 \mathrm{G}\right) .{ }^{8}$ However, the observed linewidths at $240 \mathrm{~K}$ are still broader than reported linewidths at $298 \mathrm{~K}$ due to the slower motion of radicals at lower temperature. Although the observed linewidths of the spectra (Figure S3a and b) were too large to resolve such fine features, this term was included in simulating the spectra (i.e., one electron spin $S=1 / 2$ system coupled to three nuclear spins, one nitrogen $I_{N}=1 / 2$ and two hydrogens $I_{H \beta}=I_{H \gamma}=1$ ) using spin Hamiltonian formalism. The nuclear quadrupole interaction for nitrogen nuclear spin was omitted. The Zeeman basis was chosen to express the spin operators in their matrix representations, and resonance field positions were calculated by diagonalizing $\widehat{H}$ for different $B_{0}$ values and finding the specific value of $B_{0}^{\text {res }}$ that yields the energy gap of allowed ESR transitions (i.e., $\Delta m_{s}= \pm 1$ and $m_{I}=0$ where $m_{s}$ and $m_{I}$ are electronic and nuclear spin number respectively) to match with the frequency of excitation microwave applied in the measurements. Finally, Lorentzian lineshape functions with peak-to-peak linewidth $\Gamma_{\text {Lorentzian, } p \text {-p }}$, centered at calculated resonance field positions $B_{0}^{\text {res }}$ were summed over to generate a simulated ESR spectrum for a given set of parameters: $g, \alpha_{N}, \alpha_{H}^{\beta}$, and $\alpha_{H}^{\gamma}$. Weighting the intensities of the lineshape functions for different allowed transitions according to the Boltzmann distribution as well as by the allowed transition rates were not implemented as they are negligible under the current experimental conditions. A brutal looping of the parameters ( $\Gamma_{\text {Lorentzian, } \mathrm{p}-\mathrm{p},} g, \alpha_{N}, \alpha_{H}^{\beta}$, and $\alpha_{H}^{\gamma}$ ) around the initial, educated guess was carried out to obtain the "best-simulated" spectra to that of the measurements (see Figure S3a and b). Figure S3c shows a simulated spectrum using the same parameters as those of Figure S3b but with narrower $\Gamma_{\text {Lorentzian, }}$ p-p showing the fine splitting structures from the weak hyperfine coupling to the second hydrogen. 


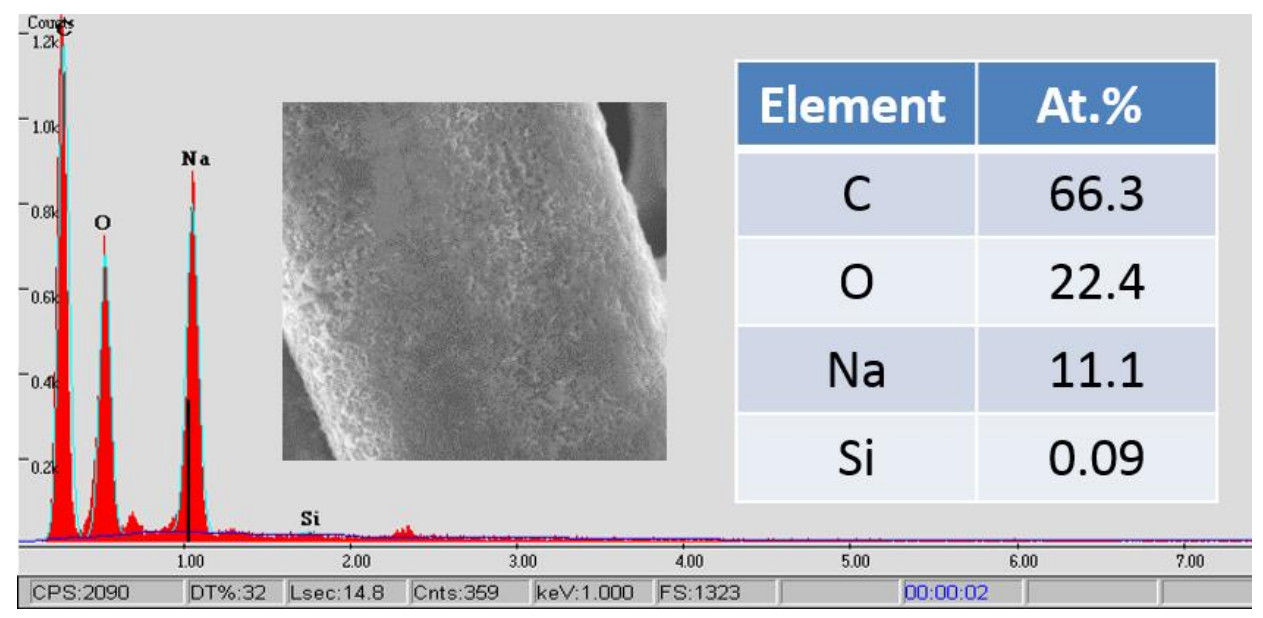

Figure S4 Morphology of film-like discharge products and the elemental composition of the discharged products analyzed by EDS.

Discussion of Figure S4 (morphology and elemental analysis of film-like products on the surface of cathodes). Figure S4 presents the morphology and elemental composition of the film-like discharge products formed at a discharge capacity of $2 \mathrm{mAh} \mathrm{cm}^{-2}$. The EDS result clearly shows a Na:O atomic ratio of $1: 2$ in the film products. It indicates that the film products are likely composed of $\mathrm{NaO}_{2}$ as previously reported ${ }^{9}$. 


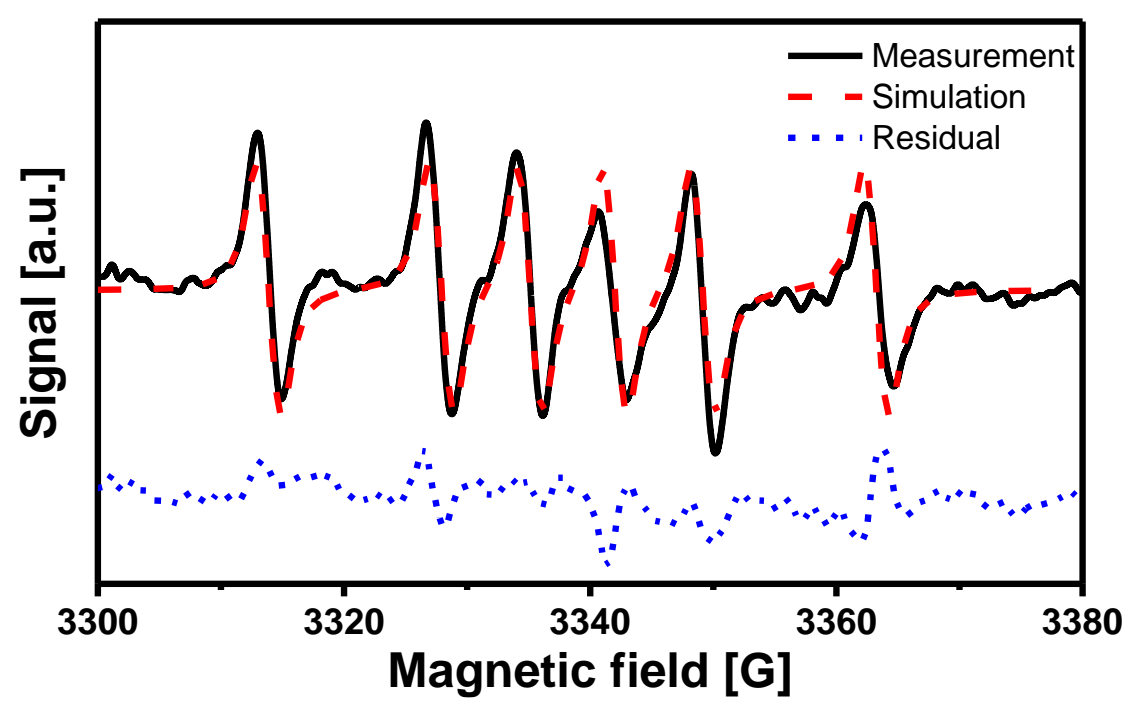

Figure S5. Measured and simulated CW ESR spectra of electrolyte sample discharged for 15 min in the presence of $5000 \mathrm{ppm} \mathrm{H}_{2} \mathrm{O}$. Black solid line indicates the measurement, red dotted line indicates the simulation, and blue dotted line indicates the residual pattern of the simulation.

Discussion of Figure S5 (ESR spectral analysis of discharged electrolyte with $5000 \mathrm{ppm} \mathrm{H}_{2} \mathrm{O}$ ). Figure S5 shows the CW ESR spectrum of electrolyte sample discharged for 15 min where 5000 ppm of $\mathrm{H}_{2} \mathrm{O}$ was added to the electrolyte. The observed spectrum is clearly different from that of DMPO-OOH radicals seen in Figure S3, mainly due to the larger hyperfine coupling strength to the hydrogen nucleus $\left(\alpha_{N}\right)$. The simulated ESR spectrum, following similar procedures described in earlier section "Confirmation of DMPO-OOH radicals by ESR spectroscopy" with simulation parameters of $g=2.0078, \alpha_{N}=14.1 \mathrm{G}$, $\alpha_{H}=21.3 \mathrm{G}, \Gamma_{\text {Lorentzian,p-p }}=1.4 \mathrm{G}$ (no hyperfine coupling to the second hydrogen nucleus spin), shows good agreement with the measurement. The hyperfine coupling strengths obtained from the simulation suggest that the paramagnetic species responsible for the observed signals are likely carbon-centered radicals, e.g., $\mathrm{CH}_{3} \mathrm{CH}_{2}{ }^{\circ}\left(\alpha_{N}=14.20 \mathrm{G}\right.$ and $\left.\alpha_{H}=20.50 \mathrm{G}\right), \mathrm{CH}_{3} \mathrm{CH}_{2} \mathrm{OCH}_{2} \mathrm{CH}_{2}{ }^{\circ}\left(\alpha_{N}=14.20 \mathrm{G}\right.$ and $\left.\alpha_{H}=20.49 \mathrm{G}\right) .^{1}$ It is worthy to note that only a limited number of carbon-centered radicals have been studied in the literature giving their hyperfine splitting constants. Thus, other carbon-centered radicals with similar structures should also be taken into account.

Recently Adams et al. concluded that glyme solvents may undergo decomposition through $\beta$-H abstraction by superoxide. ${ }^{2}$ Here, we speculate that large amounts of superoxide are formed in the presence of 5000 ppm $\mathrm{H}_{2} \mathrm{O}$. These chemically reactive superoxide radicals abstract $\beta-\mathrm{H}$ from the backbones of diglyme solvents forming $\mathrm{HO}_{2}$ and carbon-centered radicals. ${ }^{10,11}$ 


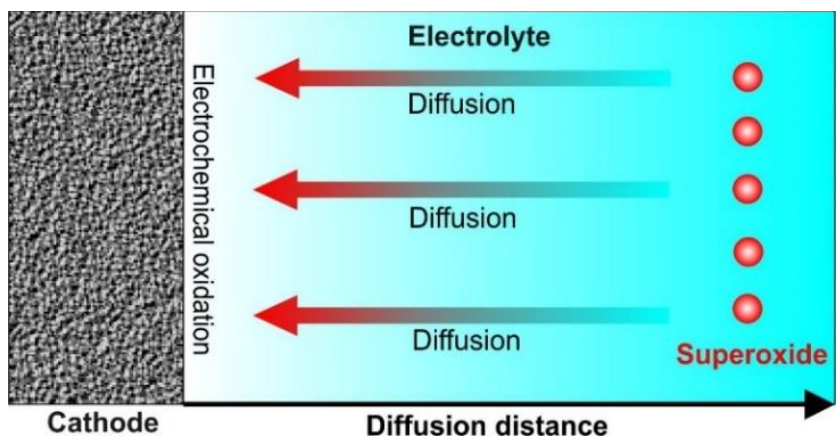

Figure S6. Model of superoxide diffusing from the electrolyte to the cathode for oxidation.

\section{Discussion of Figure S6 (solution-diffusion model).}

We assume that the charge processes simply involve the diffusion of superoxide through the electrolyte and its electrochemical oxidation on the planar cathode. This allows us to set up a non-steady state model to simulate the superoxide diffusion in the electrolyte upon charge. ${ }^{12}$ The change of superoxide concentration $(C)$ with diffusion time $(t)$ and distance $(x)$ can be described by Fick's second law as shown in eq. 1:

$$
\frac{\partial C}{\partial t}=D \frac{\partial^{2} C}{\partial x^{2}} \quad \text { eq. } 1
$$

where $D$ is the diffusion co-efficient of superoxide in the electrolyte.

After applying the Finite element analysis and simplifying eq. 1 in order to establish a discrete model, the diffusion time and distance are divided into $k$ and $j$ segments with intervals of $\Delta t$ and $\Delta x$ respectively. Thus eq. 1 can be rewritten as eq. 2 .

$$
\mathrm{C}(j, k+1)=\mathrm{C}(j, k)+\frac{D \Delta t}{\Delta x^{2}}[C(j+1, k)-2 C(j, k)+C(j-1, k)] \quad \text { eq. } 2
$$

However we need to establish initial and boundary conditions to solve eq. 2 which are given as the following: At $t=0$, the concentration of superoxide at any position in the electrolyte is equal to the solubility, $C^{*}$. (see eq. 3)

$$
\mathrm{C}(j, 0)=\mathrm{C}^{*} \quad \text { eq. } 3
$$

At $t=\Delta t$, i.e., a very short charge time, the electrochemical reaction only consumes the superoxide in the first segment of the diffusion distance, whereas no diffusion occurs in other segments. Accordingly we have the boundary condition equations, eq. 4 and 5 .

$$
\begin{aligned}
& \mathrm{C}(1,1)=\mathrm{C}^{*}-\frac{I \Delta t}{n F A \Delta x} \\
& \mathrm{C}(n, 1)=\mathrm{C}^{*}
\end{aligned}
$$

where $I$ is the charge current, $A$ is the geometric area of the cathode, $F$ is faradaic constant, and $n$ is the number of transferred electrons.

At $x=L$, i.e., at a position far from the cathode, the concentration of superoxide does not change with time, which is thereby equal to its solubility as shown in eq.6. 


$$
\mathrm{C}(j, k)=\mathrm{C}^{*} \quad \text { eq. } 6
$$

\section{Parameters}

The following parameters were used for simulation.

Table 1. Parameters used for the modelling.

\begin{tabular}{|c|c|}
\hline Parameters & Values \\
\hline Solubility $\left(C^{*}\right)$ & $0.7 \times 10^{-4} \mathrm{~mol} / \mathrm{L} *$ \\
\hline Diffusion co-efficient $(D)$ & $1.4 \times 10^{-6} \mathrm{~cm}^{2} / \mathrm{s} * *$ \\
\hline Charge current $(I)$ & $50 \mu \mathrm{A}$ \\
\hline Geometric area of cathode $(A)$ & $1 \mathrm{~cm}^{2}$ \\
\hline Charge time $(t)$ & $8000 \mathrm{~s}$ \\
\hline Diffusion distance $(L)$ & $1 \mathrm{~mm}$ \\
\hline \multicolumn{2}{|c|}{$*, * *$ the values of solubility and diffusion-efficient are estimated based on the literature. ${ }^{13,14}$} \\
\hline \multicolumn{2}{|l|}{ Results } \\
\hline \multicolumn{2}{|c|}{$\begin{array}{l}\text { If we assume that the intensity of ESR signal at a charge time of } 0 \text { min is equivalent to the solubility of } \\
\text { superoxide in diglyme (i.e., } 0.7 \times 10^{-4} \mathrm{~mol} / \mathrm{L} \text { ), the variation of the ESR intensity during charge is calculated } \\
\text { by iterating eq. } 2 \text { (see red curve in Figure } 4 \text { in the main text). Meanwhile, the effects of solubility and the } \\
\text { diffusion co-efficient on the superoxide distribution in the electrolyte were also simulated as shown below } \\
\text { in Figure S7. }\end{array}$} \\
\hline
\end{tabular}

For a charge current $(I)=50 \mu \mathrm{A}$ :
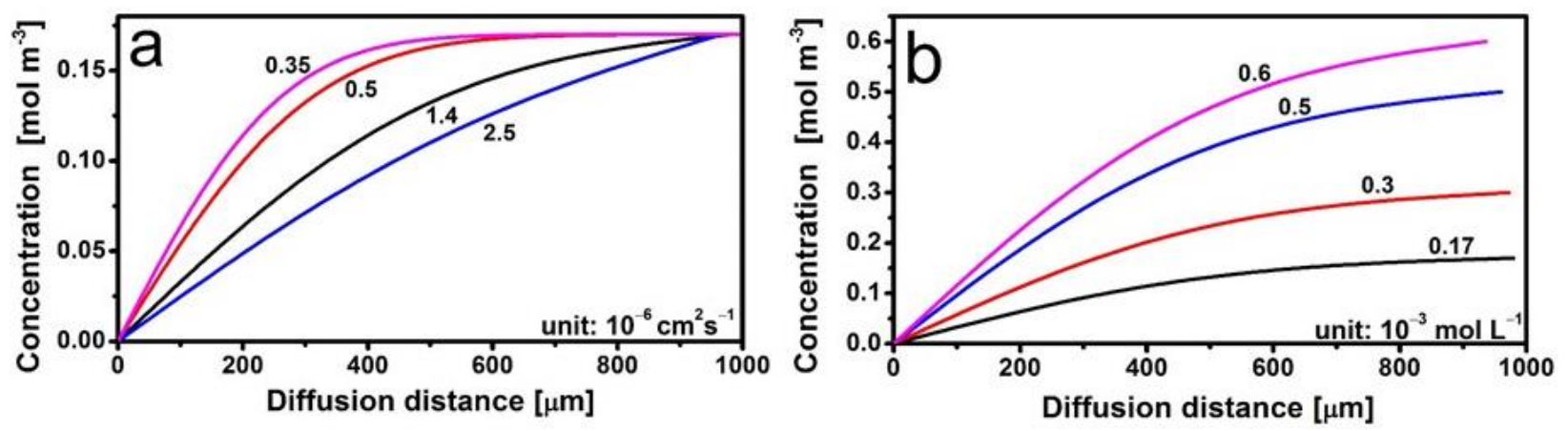

Figure S7. Superoxide distribution in the electrolyte as a function of diffusion co-efficient (a) and solubility (b) at a charge time of $600 \mathrm{~s}$. 


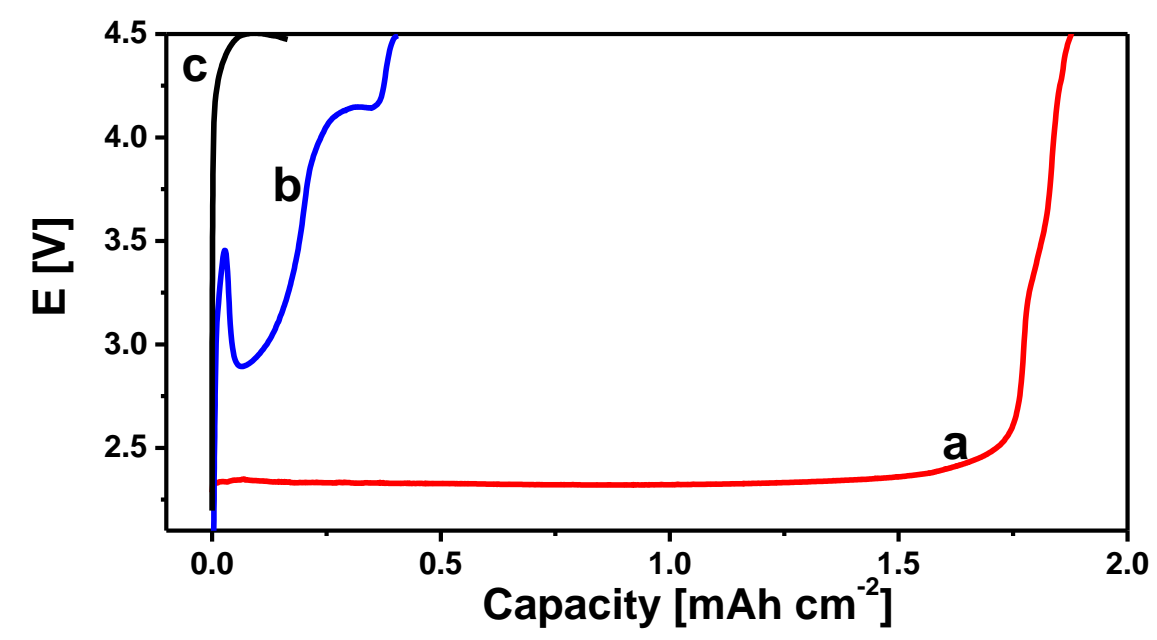

Figure S8. Comparison of charge curves of $\mathrm{Na}_{-} \mathrm{O}_{2}$ cells using diglyme (a), tetraglyme (b) and $\mathrm{Pyr}_{14} \mathrm{TFSI}$ (c) based electrolytes. Note: NaOTf was used as the conducting salt for (a) and (b), whereas sodium bis(trifluoromethanesulfonyl)imide (NaTFSI) was used for (c).

Discussion of Figure S8. To individually study the effects of solvents on the charge behavior of Na- $\mathrm{O}_{2}$ cells, $\mathrm{NaO}_{2}$ loaded electrodes were prepared by pre-discharging cathodes to $2 \mathrm{mAh} \mathrm{cm}^{-2}$ using $0.5 \mathrm{M} \mathrm{NaOTf}$ / diglyme electrolyte. The resulting electrodes were extracted from the cells, thoroughly washed with anhydrous diglyme, and dried under vacuum for $30 \mathrm{~min}$. One electrode was subjected to charge in the same electrolyte, while the other two were charged in tetraethylene glycol dimethyl ether (tetraglyme) and 1butyl-1-methylpyrrolidinium bis(trifluoromethanesulfonyl)imide ( $\left.\operatorname{Pyr}_{14} \mathrm{TFSI}\right)$ based electrolytes, respectively. As shown in Figure S8, the cell using the diglyme-based electrolyte displays a low charge plateau at $2.3 \mathrm{~V}$ with a high coulombic efficiency of $90 \%$. In contrast, the $\mathrm{Na}_{2} \mathrm{O}_{2}$ cells using tetraglyme and $\mathrm{Pyr}_{14}$ TFSI based electrolytes present much higher charge overpotentials, which deliver poor coulombic efficiencies of $20 \%$ and $5 \%$, respectively.

As described in Fick's first law (see eq. 7), the steady-state diffusion rate of superoxide in the electrolyte is determined by the superoxide solubility and diffusion co-efficient, which in turn influence the charge behaviors of $\mathrm{Na}-\mathrm{O}_{2}$ cells.

$$
J=-D \frac{\partial C}{\partial x}=-D \frac{C^{*}-C^{s}}{\Delta x} \quad \text { eq.7 }
$$

where $J$ is the diffusion rate of superoxide, $x$ is the diffusion distance, and $C^{S}$ is the concentration of superoxide at the surface of the cathode.

On the basis of RRDE studies, Gasteiger et al. reported that the diffusion coefficient of $\mathrm{O}_{2}{ }^{--}$in $\mathrm{Pyr}_{14} \mathrm{TFSI}$ is $0.11 \times 10^{-6} \mathrm{~cm}^{2} \mathrm{~s}^{-1} \cdot{ }^{14}$ In contrast, diglyme possesses a higher $D$ value of $1.4 \times 10^{-6} \mathrm{~cm}^{-2} \mathrm{~s}^{-1} .{ }^{13}$ The $D$ value in tetraglyme, to the best of our knowledge, has not been reported. However, tetraglyme shows a higher viscosity of $3.3 \times 10^{-3} \mathrm{~Pa} \bullet \mathrm{s}$ than $1.0 \times 10^{-3} \mathrm{~Pa} \bullet \mathrm{s}$ for diglyme. ${ }^{15}$ Based on the Stokes-Einstein equation, the 
theoretical $D$ value in tetraglyme is estimated to be $0.65 \times 10^{-6} \mathrm{~cm}^{2} \mathrm{~s}^{-1} \cdot{ }^{17}$ Thus, the diffusion co-efficient of superoxide follows the order of diglyme $>$ tetraglyme $>\mathrm{Pyr}_{14} \mathrm{TFSI}$.

In addition, superoxide may also present different solubilities in these solvents. Although the $C^{*}$ values of these solvents have not reported, Lee et al. recently suggested that the solvents with larger donor numbers (DN) possess higher solubility of superoxide. ${ }^{18}$ Based on their theory, we speculate that diglyme with a DN number of 19.2 show higher superoxide solubility than tetraglyme $(\mathrm{DN}=16.6)$ and pyrrolidinium based ionic liquid ( $\mathrm{DN}<10){ }^{19,20}$

Therefore, according to eq. 7, high $D$ and $C^{*}$ values of diglyme allow superoxide to readily diffuse to the cathode for oxidation, which leads to a low charge overpotential and high coulombic efficiency for Na- $\mathrm{O}_{2}$ cell as seen in Figure S8. In contrast, sluggish superoxide transfer in tetraglyme and Pyr $_{14}$ TFSI based electrolytes causes high charge overpotentials of cells with low coulombic efficiencies. 

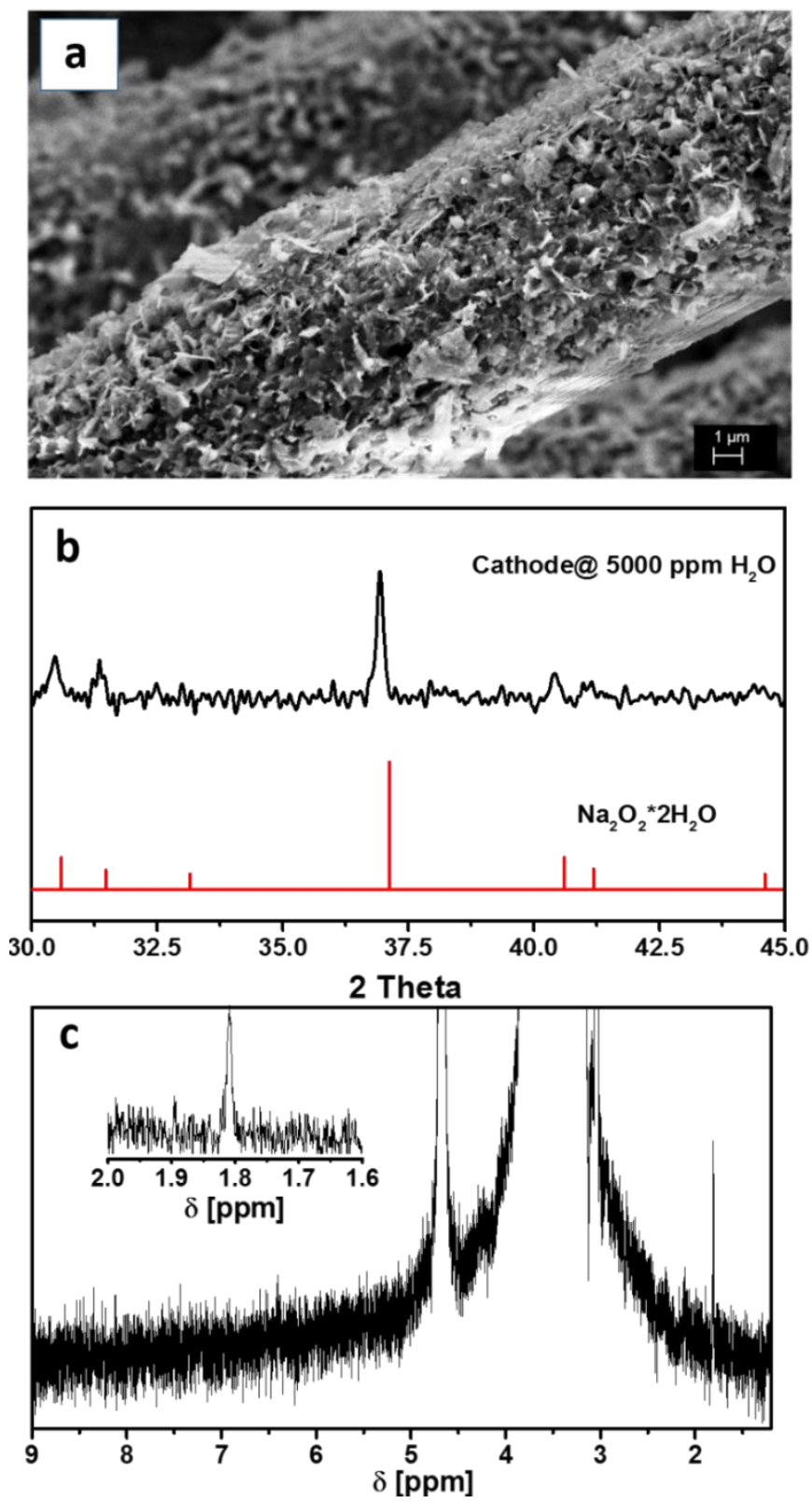

Figure S9. SEM image (a), XRD pattern (b), and ${ }^{1} \mathrm{H}-\mathrm{NMR}$ spectrum(c) of cathode after 15 min charged in the presence of $5000 \mathrm{ppm}_{2} \mathrm{O}$.

Discussion of Figure S9 (analysis of by-products formed upon charge). The cathode charged for 15 min is fully covered with flake-like particles when using electrolyte with $5000 \mathrm{ppm} \mathrm{H}_{2} \mathrm{O}$. As shown in Figure $\mathrm{S} 10 \mathrm{~b}$ and $\mathrm{c}$, the XRD pattern and ${ }^{1} \mathrm{H}-\mathrm{NMR}$ spectrum of this cathode prove that these surface particles are composed of crystalline $\mathrm{Na}_{2} \mathrm{O}_{2} \cdot 2 \mathrm{H}_{2} \mathrm{O}$ and amorphous sodium acetate which gives a NMR peak at $\sim 1.8$ ppm. ${ }^{21,} 22$ 


\section{References for Supporting Information}

(1) Buettner, G. R. Free Radic. Biol. Med. 1987, 3 (4), 259.

(2) Adams, B. D.; Black, R.; Williams, Z.; Fernandes, R.; Cuisinier, M.; Berg, E. J.; Novak, P.; Murphy, G. K.; Nazar, L. F. Adv. Energy Mater. 2015, 5 (1), n/a.

(3) Lu, J.; Jung, H.-J.; Lau, K. C.; Zhang, Z.; Schlueter, J. A.; Du, P.; Assary, R. S.; Greeley, J.; Ferguson, G. A.; Wang, H.-H.; Hassoun, J.; Iddir, H.; Zhou, J.; Zuin, L.; Hu, Y.; Sun, Y.-K.; Scrosati, B.; Curtiss, L. A.; Amine, K. ChemSusChem 2013, 6 (7), 1196.

(4) Rosen, G. M.; Rauckman, E. J. Mol. Pharmacol. 1980, 17 (2), 233.

(5) Farmer, P. J.; Gidanian, S.; Shahandeh, B.; Di Bilio, A. J.; Tohidian, N.; L. Meyskens, F. Pigment Cell Res. 2003, 16 (3), 273.

(6) Harbour, J. R.; Hair, M. L. J. Phys. Chem. 1978, 82 (12), 1397.

(7) Eastland, G. W.; Symons, M. C. R. J. Phys. Chem. 1977, 81 (15), 1502.

(8) Buettner, G. R. Free Radic. Biol. Med. 1987, 3 (4), 259.

(9) Xia, C.; Black, R.; Fernandes, R.; Adams, B.; Nazar, L. F. Nat. Chem. 2015, 7 (6), 496.

(10) Andrieux, C. P.; Hapiot, P.; Saveant, J. M. J. Am. Chem. Soc. 1987, 109 (12), 3768.

(11) Sawyer, D. T.; Roberts, J. L.; Calderwood, T. S.; Sugimoto, H.; McDowell, M. S.; Wardman, P. Philos. Trans. R. Soc. B Biol. Sci. 1985, 311 (1152), 483.

(12) Bard, A. J.; Faulkner, L. R. Electrochemical methods: fundamentals and applications, 2nd ed.; Wiley: New York, 2001.

(13) Hartmann, P.; Heinemann, M.; Bender, C. L.; Graf, K.; Baumann, R.-P.; Adelhelm, P.; Heiliger, C.; Janek, J. J. Phys. Chem. C 2015, 119 (40), 22778.

(14) Herranz, J.; Garsuch, A.; Gasteiger, H. A. J. Phys. Chem. C 2012, 116 (36), 19084.

(15) Pal, A.; Singh, Y. P. J. Chem. Eng. Data 1996, 41 (5), 1008.

(16) Hartmann, P.; Grübl, D.; Sommer, H.; Janek, J.; Bessler, W. G.; Adelhelm, P. J. Phys. Chem. C 2014, $118(3), 1461$.

(17) Read, J.; Mutolo, K.; Ervin, M.; Behl, W.; Wolfenstine, J.; Driedger, A.; Foster, D. J. Electrochem. Soc. 2003, 150 (10), A1351.

(18) Johnson, L.; Li, C.; Liu, Z.; Chen, Y.; Freunberger, S. A.; Ashok, P. C.; Praveen, B. B.; Dholakia, K.; Tarascon, J.-M.; Bruce, P. G. Nat. Chem. 2014, 6 (12), 1091.

(19) Dany Brouillette; Gérald Perron; Jacques E. Desnoyers. J. Solut. Chem. 27 (2), 151.

(20) Tachikawa, N.; Yoshii, K.; Katayama, Y. Bunseki Kagaku 2015, 64 (3), 211.

(21) Zhao, N.; Li, C.; Guo, X. Phys. Chem. Chem. Phys. 2014, 16 (29), 15646.

(22) Bi, X.; Ren, X.; Huang, Z.; Yu, M.; Kreidler, E.; Wu, Y. Chem Commun 2015, 51 (36), 7665. 